\title{
An exploration of the role of pharmacists within general practice clinics: the protocol for the pharmacists in practice study (PIPS)
}

\author{
Edwin Tan ${ }^{1}$, Kay Stewart ${ }^{1}$, Rohan A Elliott ${ }^{1,2}$ and Johnson George ${ }^{1 *}$
}

\begin{abstract}
Background: Medication-related problems are a serious concern in Australian primary care. Pharmacist interventions have been shown to be effective in identifying and resolving these problems. Collaborative general practitioner-pharmacist services currently available in Australia are limited and underused. Limitations include geographical isolation of pharmacists and lack of communication and access to patient information. Co-location of pharmacists within the general practice clinics is a possible solution. There have been no studies in the Australian setting exploring the role of pharmacists within general practice clinics.

The aim of this study is to develop and test a multifaceted practice pharmacist role in primary care practices to improve the quality use of medicines by patients and clinic staff.

Methods/design: This is a multi-centre, prospective intervention study with a pre-post design and a qualitative component. A practice pharmacist will be located in each of two clinics and provide short and long patient consultations, drug information services and quality assurance activities. Patients receiving long consultation with a pharmacist will be followed up at 3 and 6 months. Based on sample size calculations, at least 50 patients will be recruited for long patient consultations across both sites. Outcome measures include the number, type and severity of medication-related problems identified and resolved; medication adherence; and patient satisfaction. Brief structured interviews will be conducted with patients participating in the study to evaluate their experiences with the service. Staff collaboration and satisfaction with the service will be assessed.

Discussion: This intervention has the potential to optimise medication use in primary care clinics leading to better health outcomes. This study will provide data about the effectiveness of the proposed model for pharmacist involvement in Australian general practice clinics, that will be useful to guide further research and development in this area.
\end{abstract}

Trial registration: Australian New Zealand Clinical Trials Registry: ACTRN12612000742875

Keywords: Pharmacists, Primary healthcare, General practice, Multidisciplinary, Family practice

\section{Background}

Medication misadventure remains a serious concern in Australian primary care $[1,2]$. It is estimated that one in 10 patients who visit their general practitioner (GP) experience a medication-related problem (MRP), of which almost half are considered moderate or severe,

\footnotetext{
* Correspondence: johnson.george@monash.edu

${ }^{1}$ Centre for Medicine Use and Safety, Faculty of Pharmacy and Pharmaceutical Sciences, Monash University, 381 Royal Parade, Parkville, VIC 3052, Australia

Full list of author information is available at the end of the article
}

with $8 \%$ requiring hospitalization [1]. Approximately one in four of these are preventable. These data are consistent with studies from other countries. For example, a recent UK study revealed that one in 20 prescription items in general practice contained an error, affecting 1 in 8 patients [3]. Poor communication has been identified as a major contributing factor towards MRPs, [2] highlighting the need for greater collaboration between GPs, pharmacists and other primary health professionals to ensure optimal patient care.

Collaborative medication reviews, undertaken by pharmacists and GPs, have been successful in identifying and

\section{Ciomed Central}


resolving medication-related problems, improving quality of prescribing, and optimizing drug use and costs $[4,5]$. These collaborative services, however, are currently limited and underused due to factors including geographical isolation, limited access to patient medical records, a lack of time for team activities and a health policy that is not conducive to such collaborative arrangements [6]. Additionally, communication between GPs and pharmacists in the community setting is sporadic and reactive, risking fragmentation of patient care [7].

A possible solution to these problems is the integration of pharmacists into general practices and primary healthcare clinics. In countries such as the United Kingdom, Canada and the United States, practice pharmacists work in close collaboration with GPs (family physicians) to undertake a range of clinical and administrative tasks $[8,9]$. Some studies suggest the implementation of these roles can result in improved medication use and health outcomes and reduced health service use and cost [10-12]. Co-location has also been shown to enable greater communication and cooperation between health professionals, and strengthen the primary health care team [13].

The aim of this study is to develop and test a multifaceted practice pharmacist role in primary care practices in Australia to improve the quality use of medicines by patients and clinic staff.

\section{Methods/design}

This is a multi-centre, prospective intervention study with a pre-post design and a qualitative component, involving two general practice (primary care) clinics in Melbourne, Australia.

\section{Recruitment of practices and pharmacists}

Primary care clinics will be invited to participate through advertisements and consultation with the Victorian Divisions of General Practice and key informants in Melbourne, Australia. Primary care practices that have the space to accommodate a co-located pharmacist will be targeted. An independent practice pharmacist with significant clinical experience and accreditation to conduct Government funded collaborative Home Medicines Reviews (HMRs) will be identified for each clinic through advertisements and key informants.

\section{Intervention}

The intervention will consist of a multi-faceted, collaborative service targeting patients and practice staff. A practice pharmacist will be co-located in each of the study clinics for at least eight hours per week for six months. The practice pharmacist will undertake the following tasks:

1. Long patient consultations

2. Short patient consultations
3. Drug information and education service for clinic staff; and

4. Quality assurance activities

\section{Long patient consultation}

Recruitment of participants

Participants for the long patient consultations will be practice patients who may be at an increased risk of MRPs [14].

\section{Inclusion criteria}

- Using five or more medicines

- Using one or more medicines that require therapeutic drug monitoring (e.g. warfarin, phenytoin, lithium)

- Using medicines for three or more medical problems

- Have had a recent unplanned hospital admission/ emergency department visit

- Having other reason(s) for being at risk of medication misadventure (e.g. adherence issues, language barriers, multiple prescribers)

\section{Exclusion criteria}

- Have had a HMR in the previous 12 months with no subsequent significant change in clinical status or medication regimen

- Are unable to provide written informed consent

- Are under 18 years of age

- Are unavailable for follow up for six months after recruitment

Patients meeting one or more of the eligibility criteria will be considered for referral to the study by their general practitioner or clinic staff. Those patients who are referred to the study will be provided with an introductory letter and plain language statement in person during their clinic visit. Patients will be asked by the GP/clinic staff if they are willing to be contacted by the research team. If patients agree, the GP/clinic staff will provide the research team with the patient's contact details.

The research assistant will contact patients who agreed to provide their contact details. Suitable and willing participants will be recruited by obtaining initial verbal consent. Written consent will be obtained at the time of the appointment with the practice pharmacist.

\section{Baseline data collection}

Baseline data will be collected by the research assistant using a structured questionnaire, either in person (at the clinic or the person's home) or by telephone, and will include demographic information (age, sex, ethnicity, 
education, socioeconomic status, living arrangements etc.), health information (general health, health service use, health literacy [15] etc.), and medicines information (medication risk [14] adherence [16,17] etc.). The research assistant will then organize for the participant to meet the practice pharmacist for a long consultation.

\section{Long patient consultation process}

All participants will receive a 30-60 minute consultation with the pharmacist in a private room at the clinic (or a home visit if they prefer or are housebound) to perform a comprehensive medication review and identify medication-related problems (MRPs). Prior to the consultation, the pharmacist will discuss any health or medication-related issues with the GP or clinic staff, if needed. The pharmacist will also review participants' general practice medical record (including progress notes, medication lists and pathology results) and dispensing histories if needed. The pharmacist will obtain written informed consent from the participant. In addition to reviewing the participant's medication regimen, the pharmacist will assess medication adherence and knowledge. The pharmacist will provide counseling and education as needed on medication management and the use of medication devices, and reinforce lifestyle advice related to their health problems and medications. The pharmacist may provide the participant with a complete medication list and refer them to their community pharmacy for adherence aids (e.g. pill boxes, administration aids), if needed. Additionally, referral may be made to the GP or other health professionals as required. After the consultation, the pharmacist will write a short report outlining MRPs and recommendations, and provide this to the participant's GP either electronically (via secure email or directly into the practice's electronic medical record) or as a paper-based report, depending on the GP's preference. The pharmacist may update the medication history within the practice's medical record as needed. Following this, the pharmacist will discuss any issues with the GP, other staff and community pharmacist, if needed. Case conferencing may be organized where appropriate.

\section{Monitoring and follow up}

Participants will be followed up by the research assistant at three and six months either in the clinic or by telephone to collect data about implementation of pharmacist recommendations, resolution of MRPs identified by the practice pharmacist, participant's medication adherence and participant's general health and wellbeing.

\section{Sample size}

Based on an expected average number of 2.5 MRPs per participant at baseline, an expected average reduction of 1 MRP (i.e. 40\%) per participant with a withinparticipant standard deviation of 2.1 MRPs (assuming a correlation coefficient of 0.5$),[18,19]$ a power of $80 \%$ and a two-tailed alpha of 0.05 , the required sample size is 37 participants. Allowing for a dropout/loss to followup rate of $25 \%$, at least 50 participants will be recruited. This was calculated using PS Power and Sample Size Calculations (Version 3.0, Dupont \& Plummer, 2009).

\section{Short patient consultation}

Patients with potential medication issues (e.g. nonadherence, newly prescribed medicines) who do not require a comprehensive review of their complete medication regimen may receive a short consultation with the pharmacist. Data will be collected about these consultations, but the patients will not be consented for the study (no personal or identifying data will be collected). Referrals may be made by GPs or clinic staff, or patients may self-refer. The short patient consultation will last 15-30 minutes and provides an opportunity for the pharmacist to provide brief education and counseling on specific needs or answer questions. Short patient consultations will only be undertaken in the clinic. After the consultation, the pharmacist will write brief notes directly into the electronic medical record, and update records as needed. Examples of services to be provided in these consultations include: new medication counseling, adherence assessment, assessment of and education on device technique (e.g. using asthma inhalers), and provision of a medication list. Patient satisfaction will be assessed by an anonymous questionnaire to be given to each patient by the pharmacist along with a reply-paid envelope addressed to the researchers.

\section{Drug information and education service}

The practice pharmacist will provide a drug information service for practice staff. Queries can be made to the pharmacist in person, by telephone or via email. All queries (and responses) will be documented by the pharmacist. Practice staff will also be invited to attend pharmacist-led group education sessions targeting topics relevant to them (e.g. new therapy or treatment protocols), and a weekly drug information newsletter may also be produced by the pharmacist and provided to staff.

\section{Quality assurance activities}

The practice pharmacists will undertake a Drug Use and Evaluation (DUE) program in the clinic. The pharmacists will review current prescribing patterns, evaluate these against current best practice guidelines, and implement an intervention to address deficiencies identified using established DUE methodology [20]. A pharmacotherapeutic area of concern or importance will be identified by the pharmacist in conjunction with the GPs 
and clinic staff. The practice team, with assistance from the research team, will decide upon audit criteria and measurement instruments will be derived from published clinical practice guidelines. The pharmacist will collect and evaluate data relevant to medication use in this area by retrospectively reviewing electronic medical records. Confidentiality of data will be ensured by de-identifying all information collected. Results and feedback will be provided to staff. Multifaceted strategies to improve the quality of prescribing in the selected area will be developed and implemented by the interdisciplinary team, and re-evaluated at the end of the study period. Patients will not be recruited or consented to this part of the study, and no identifying data will be collected.

\section{Monitoring and follow up}

Actions arising from the DUE program (e.g. development and implementation of clinical guidelines, criteria, treatment protocols, education programs) will be assessed for effectiveness after six months by repeating the prescribing audit to complete the DUE cycle [20].

\section{Outcomes}

\section{Outcomes of long patient consultations}

Medication-related problems The primary outcome of the long consultations will be the number of MRPs identified by practice pharmacists and the number of identified MRPs that are resolved as a result of the pharmacists' interventions. The number of MRPs, number of recommendations made by the pharmacists and the number of recommendations accepted/implemented will be recorded and determined by chart audit and/or patient interview, conducted by a research assistant. The types of problems will be categorized by the research team according to the criteria described by Strand et al [21]. The severity of the MRPs and the likely consequences if they had not been addressed will be assessed by an expert panel using a risk classification system [22].

Medication adherence Participants' medication adherence will be measured using two methods - the Morisky Scale [16] and the Tool for Adherence Behaviour Screening (TABS) [17] at baseline, three and six months. The Morisky Scale is a validated 4-item scale that asks patients four 'yes/no' questions regarding patterns of medication use. A patient is considered non-adherent if they answer 'yes' to any of the questions. The TABS is a validated and reliable sub-scale of the Beliefs and Behaviour Questionnaire (BBQ). The TABS consists of two, 4-item subscales for adherence and non-adherence. It screens for medication non-adherence that is both intentional and unintentional and assesses the respondent's agreement with a series of statements that are scored on a five-point Likert scale. The total score for 'nonadherence' will be subtracted from that of 'adherence'; a differential of $\geq 15$ will be considered as good adherence and $\leq 14$ will be considered as suboptimal adherence [23].

General health Patient general health and wellbeing, including use of health services, will be assessed via patient self-report at baseline, three and six months.

\section{Other outcomes \\ Satisfaction}

Patient satisfaction with the practice pharmacist will be determined by a structured, satisfaction questionnaire adapted from a previously validated patient satisfaction survey by Baker regarding physician consultations $[24,25]$. The questionnaire will be anonymous and provided at the end of any interaction with the pharmacist (including long patient consultations and short patient consultations). Participants will be requested to complete and return the questionnaire before they leave the practice or at their earliest convenience, using a replypaid envelope.

\section{Quality of prescribing and medication use}

The effectiveness of the DUE program will be assessed by re-evaluating medication use in the targeted population at the end of the study and comparing this to baseline data.

\section{Drug information queries}

The number of drug information queries made and answered, the type of queries made and by whom, will be evaluated at six months.

\section{Short patient consultations}

General information regarding the nature of the short consultations will be recorded, including the reason for referral, type of service provided and average time spent.

\section{Experiences and feedback}

The views of a sample of stakeholders on their experiences with this new service will be explored using interviews and/or focus groups at the study's conclusion. Patients and staff will have the opportunity to share their thoughts on the perceived benefits and challenges of the service and how it could be improved and developed further.

\section{Data analysis}

Data will be entered into the Statistical Package for Social Sciences (SPSS) for Windows Version 19.0 (IBM, New York, USA) and analyzed using standard descriptive methods. Bivariate analysis will be performed 
between pre- and post-intervention data, using paired t-tests for continuous variables, McNemar chi-square tests for categorical variables, and Wilcoxon matchedpairs signed-ranks tests for ordinal variables. Comparisons between practices may be done using repeated measures analysis of variance (ANOVA). Binary logistic regression may be performed to identify independent predictors of having medication-related problems and effects on adherence and other outcomes.

Qualitative data management will be facilitated using NVivo ${ }^{\circledR}$ (Version 9, Qualitative Solutions \& Research International, Melbourne, Vic). Interview transcripts will be read by two independent researchers and coded for emergent themes. Any discrepancies will be discussed and sorted in team meetings in the presence of a third researcher. A framework approach may be utilized whereby a thematic framework, based on a priori issues, will be applied to the data [26]. This will allow data to be easily indexed and charted, thus aiding subsequent interpretation.

\section{Ethics}

This study has been approved by the Monash University Human Research Ethics Committee.

\section{Discussion}

To the best of our knowledge, this is the first study in Australia to evaluate an interdisciplinary, multifaceted practice pharmacist role to improve the quality use of medicines by both patients and clinic staff. The comprehensive nature of this intervention aims to optimise medicine use at several levels. Although the study will be conducted with a pre-post design, subjects will serve as their own controls, thus eliminating inter-subject variability and reducing confounding. Additionally, the study will be conducted in more than one primary care clinic allowing for inter-practice comparison and improving external validity.

The study involves mixed methods to capture and measure a variety of data to explain the impact of the intervention. Additionally, a range of process, health, medication management and humanistic outcomes will be collected. By using both quantitative and qualitative methods, a deeper exploration of the intervention can occur. This will enable a more comprehensive exploration of the various intricacies involved in both types of settings, thus allowing identification of the optimal models of pharmacist integration for various primary care clinics.

\section{Limitations}

The before and after design, which lacks a concurrent control group, may compromise the internal validity of the study and limit the conclusions drawn from the results. Non-random sampling means external validity is also compromised. However, this design is the most suitable and practical given the nature of the intervention; a controlled trial with randomization at the level of the patient would be severely limited by contamination, and a cluster randomized controlled trial would require multiple practices, which is not feasible in the context of limited resources.

\section{Conclusion}

The integration and co-location of a practice pharmacist into Australian primary healthcare clinics is uncommon and has not been evaluated. This study will implement and evaluate a new collaborative pharmacist role and assess its effects on optimizing medication outcomes at various levels. The study will provide useful data to guide further research and development in this area.

\section{Abbreviations}

MRP: Medication-related Problem; GP: General Practitioner; HMR: Home Medicines Review; DUE: Drug Use Evaluation; TABS: Tool for Adherence Behaviour Screening; BBQ: Beliefs and Behaviour Questionnaire.

\section{Competing interests}

The authors declare that they have no competing interests.

\section{Authors' contributions}

ET (PhD candidate) participated in the design of the trial, recruitment and manuscript preparation, and has an ongoing role in carrying out the trial. JG, RAE and KS participated in the design of the trial and study methodology, and review of the manuscript. All authors read and approved the final manuscript.

\section{Acknowledgements}

The project is funded by the Windermere Foundation.

\section{Author details}

${ }^{1}$ Centre for Medicine Use and Safety, Faculty of Pharmacy and Pharmaceutical Sciences, Monash University, 381 Royal Parade, Parkville, VIC 3052, Australia. ${ }^{2}$ Pharmacy Department, Austin Health, Studley Rd, Heidelberg, VIC 3084, Australia.

Received: 29 June 2012 Accepted: 29 July 2012 Published: 9 August 2012

\section{References}

1. Miller GC, Britth HC, Valenti L: Adverse drug events in general practice patients in Australia. Med J Aust 2006, 184:321-324.

2. Bhasale AL, Miller GC, Reid SE, Britt HC: Analysing potential harm in Australian general practice: an incident-monitoring study. Med J Aust 1998, 169:73-76.

3. Avery T, Barber N, Ghaleb M, Dean Franklin B, Armstrong S, Crowe S, Dhillon S, Freyer A, Howard R, Pezzolesi C, et al: Investigating the prevalence and causes of prescribing errors in general practice: the PRACtICe study. Gen Med Council 2012.

4. Gilbert AL, Roughead EE, Beilby J, Mott K, Barratt JD: Collaborative medication management services: improving patient care. Med J Aust 2002, 177:189-192.

5. Stafford L, Stafford A, Hughes J, Angley M, Bereznicki L, Peterson G: Drug-related problems identified in post-discharge medication reviews for patients taking warfarin. Int J Clin Pharm 2011, 33:621-626.

6. Edmunds J, Calnan MW: The reprofessionalisation of community pharmacy? An exploration of attitudes to extended roles for community pharmacists amongst pharmacists and General Practioners in the United Kingdom. Soc Sci Med 2001, 53:943-955. 
7. Farris K, Cote I, Feeny D, Johnson J, Tsuyuki R, Brilliant S, Dieleman S: Enhancing primary care for complex patients. Demonstration project using multidisciplinary teams. Can Fam Physician 2004, 50:998-1003.

8. Silcock J, Raynor DKT, Petty D: The organisation and development of primary care pharmacy in the United Kingdom. Health Policy 2004, 67:207-214.

9. American Society of Health-system Pharmacists: ASHP Statement on the Pharmacist's Role in Primary Care. Am J Health-Sys Ph 1999, 56:1665-1667.

10. Dolovich L, Pottie K, Kaczorowski J, Farrell B, Austin Z, Rodriguez C, Gaebel $\mathrm{K}$, Sellors C: Integrating family medicine and pharmacy to advance primary care therapeutics. Clin Pharmacol Ther 2008, 83:913-917.

11. Borenstein JE, Graber G, Saltiel E: Physician-Pharmacist Comanagement of Hypertension: A Randomized, Comparative Trial. Pharmacotherapy 2003, 23:209-216

12. Zermansky AG, Petty DR, Raynor DK, Freemantle N, Vail A, Lowe CJ: Randomized controlled trial of clinical medication review by a pharmacist of elderly patients receiving repeat prescriptions in general practice. Brit Med J 2001, 6:1340-1343.

13. Farrell B, Pottie K, Woodend K, Yao V, Dolovich L, Kennie N, Sellors C: Shifts in expectations: Evaluating physicians' perceptions as pharmacists become integrated into family practice. J Interprof Care 2010, 24:80-89.

14. Barenholtz Levy H: Self-administered medication-risk questionnaire in an elderly population. Ann Pharmacother 2003, 37:982-987.

15. Chew LD, Bradley KA, Boyko EJ: Brief questions to identify patients with inadequate health literacy. Fam Med 2004, 36:588-594.

16. Morisky DE, Green LW, Levine DM: Concurrent and predictive validity of a self-reported measure of medication adherence. Med Care 1986, 24:67-74.

17. George J, Mackinnon A, Kong DC, Stewart K: Development and validation of the Beliefs and Behaviour Questionnaire (BBQ). Patient Educ Couns 2006, 64:50-60.

18. Sellors J, Kaczorowski J, Sellors C, Dolovich L, Woodward C, Willan A, Goeree R, Cosby R, Trim K, Sebaldt R, et al: A randomized controlled trial of a pharmacist consultation program for family physicians and their elderly patients. Can Med Assoc J/ 2003, 169:17-22.

19. Soendergaard B, Kirkeby B, Dinsen C, Herborg H, Kjellberg J, Staehr P: Drugrelated problems in general practice: results from a development project in Denmark. Pharm World Sci 2006, 28:61-64.

20. SHPA Committee of Specialty Practice in Drug Use Evaluation: SHPA Standards of Practice for Drug Use Evaluation in Australian Hospitals. J Pharm Pract and Res 2004, 34:220-223.

21. Strand LM, Morely PC, Cipolle RJ, Ramsey R, Lamsam GD: Drug-related problems: their structure and function. Ann Pharmacother 1990, 24:1093-1097.

22. Elliott R, Woodward M: Assessment of risk associated with medicationrelated problems in elderly outpatients. J Pharm Pract and Res 2009, 39:109-113.

23. Lau R, Stewart K, McNamara KP, Jackson SL, Hughes JD, Peterson GM, Bortoletto DA, McDowell J, Bailey MJ, Hsueh A, George J: Evaluation of a community pharmacy-based intervention for improving patient adherence to antihypertensives: a randomized controlled trial. $B M C$ Health Serv Res 2010, 10:34.

24. Baker R: Development of a questionnaire to assess patients' satisfaction with consultations in general practice. Br J Gen Pract 1990, 40:487-490.

25. Stewart DC, George J, Bond CM, Cunningham IT, Diack HL, McCaig DJ: Exploring patients' perspectives of pharmacist supplementary prescribing in Scotland. Pharm World Sci 2008, 30:892-897.

26. Pope C, Ziebland S, Mays N: Qualitative research in health care. Analysing qualitative data. Brit Med J 2000, 320:114-116.

doi:10.1186/1472-6963-12-246

Cite this article as: Tan et al: An exploration of the role of pharmacists within general practice clinics: the protocol for the pharmacists in practice study (PIPS). BMC Health Services Research 2012 12:246.

\section{Submit your next manuscript to BioMed Central and take full advantage of:}

- Convenient online submission

- Thorough peer review

- No space constraints or color figure charges

- Immediate publication on acceptance

- Inclusion in PubMed, CAS, Scopus and Google Scholar

- Research which is freely available for redistribution

Submit your manuscript at www.biomedcentral.com/submit
Ciomed Central 